\title{
Management of Hydatid Cyst of the Spleen. A Case Report.
}

Azzi. Nadjet, Yakoubi. Becherki *,

Department of University Hospital of Bab el Oued Algiers- Algeria.

*Corresponding author: Yakoubi. Becherki, Department of University Hospital of Bab el Oued Algiers- Algeria. E-mail: becherky@ yahoo.fr Received Date: February 2, 2020; Accepted Date: February 5, 2020; Published Date: February 17, 2020.

Citation:Nadjet A, Becherki Y. (2020) Management of Hydatid Cyst of the Spleen. A Case Report. Journal of Clinical Case Reports and Studies, 1(1): DOI: $10.31579 /$ ccrs.2020/001

Copyright: @ 2020. Yakoubi. Becherki. This is an open-access article distributed under the terms of the Creative Commons Attribution License, which permits unrestricted use, distribution, and reproduction in any medium, provided the original author and source are credited.

\begin{abstract}
Echinococcosis (hydatid disease) primarily affects the liver; however, secondary involvement due to hematogenous dissemination may be seen in almost any anatomic location. Isolated hydatid disease of the spleen is rare $(1,2)$. It is caused by the larval form of the tapeworm Echinococcus granulosus, E. multilocularis, E. vogeli, or E. oligarthrus. E. granulosus is the most common organism involved, with dogs as the definitive host and sheep as an intermediate host. Human beings exposed to certain stages of the life cycle of the organism are also an intermediate host. Human hydatid disease can involve the liver (55\%-70\%), lung (18\%-35\%), spleen, kidney, peritoneal cavity, skin and muscles $(<2 \%)$ and rarely the remaining parts of the body.
\end{abstract}

Keys Words: echinoccocus granulosus; splenic hydatid; laparotomy

\section{Introduction}

Splenic hydatid disease is very rare with its occurrence less than $3 \%$ of the total incidence of Echinococcosis even in endemic areas.

Berlot first described splenic hydatid cyst as an autopsy finding in 1790 . Primary infestation of the spleen usually takes place by the arterial route after the parasite has passed the two filters (hepatic and pulmonary).

We report a case of a primary isolated splenic hydatid cyst treated with a classical surgical approach. This case report and literature review describes the management of hydatid splenic invasion.

\section{Presentation of Case}

We report below the case of a 28 year old male; originally from Ain Defla (Fig. 1) and living there, without any particular history; in whom the discovery of this pathology was fortuitous during the exploration of a urinary symptomatology (lumbar pain).Symptomatology (lumbar pain).

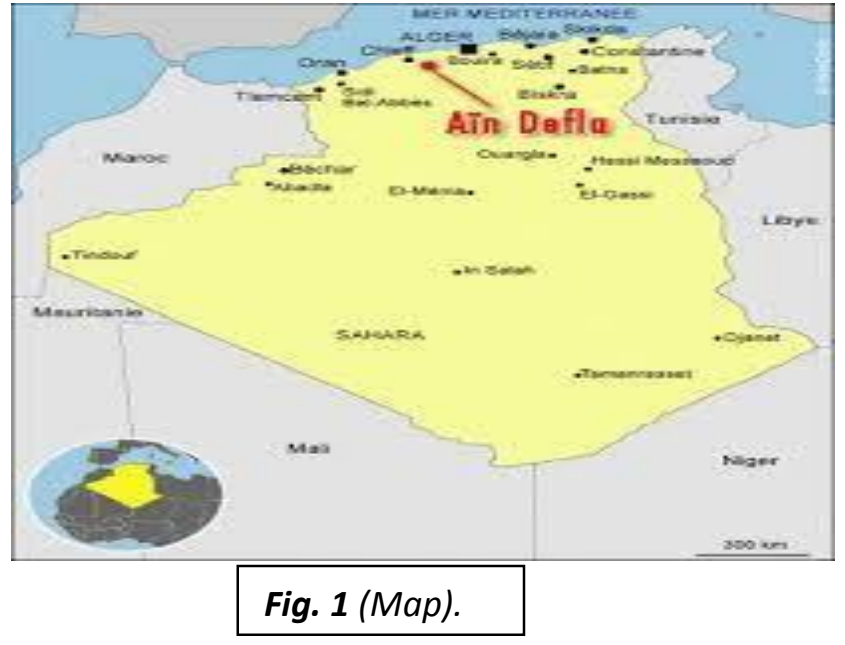

Physical examination showed an asymetric abdomen and a growing lump with smooth surface in left hypochondriac, epigastric and umbilical region.

Mild epigastric tenderness was noted with no rebound tenderness, guarding, or hepatomegaly. There was no lymphadenopathy.

Chest, cardiovascular, central nervous, and the musculoskeletal systems were normal on examination.

Routine laboratory investigation (coagulation profile, biochemistry, renal function test, liver function test and electrolytes) revealed no abnormalities.

The diagnosis was confirmed by a serological test.

Abdominal ultrasonography and computed tomography (CT) Fig. 2 revealed a cyst located in the spleen ; that one notes a voluminous hydatid cyst and showed round, well defined, cystic lesion large cystic lesion in spleen measuring $30 \times 25 \mathrm{~cm}$ over pancreas which moving left kidney and spleen and displacing the stomach and the intestines to the right .

The cyst in the spleen appeared to fill the left quadrant of the abdominal cavity.

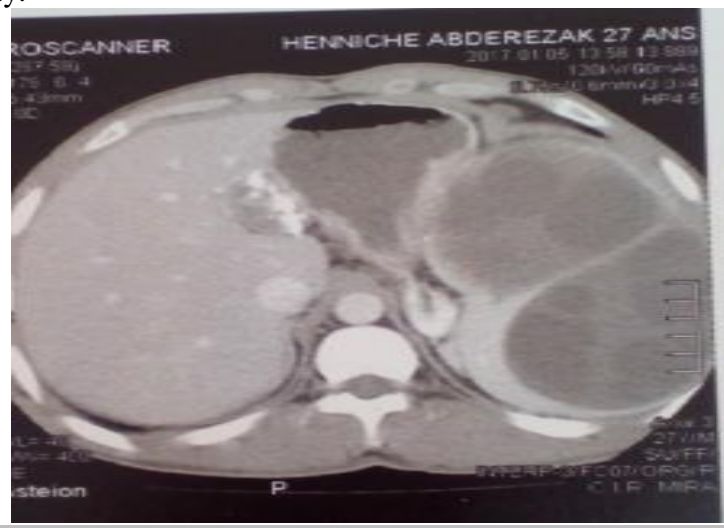

Figure. 2 Computed tomography scan revealing a Giant cyst in the spleen 
Laparotomy was performed through a midline incision. Surgical exploration revealed a hydatid cyst occupying whole splenic parenchyma. The mass measuring approximately $300 \times 200 \mathrm{~mm}$ was attached to left diaphragm and the stomach Fig.3, 4. The rest of the abdominal organs were normal. The abdomen was washed locally with peroxide hydrogen Peroxide (H2O2). The surgical intervention was radical as splenectomy carrying the cyst with difficulties because the latter had relations with the kidney, the stomach and the diaphragm Fig. 4. On cut section, there was hydatid sand and fluid around four liters and daughter's cysts Fig.6.

Total removal of the cyst with the spleen Fig.7. The postoperative period was uneventful and the patient was discharged on the postoperative day 7. The clinical and ultrasonography follow-up did not show any evidence of recurrence at six months. After the splenectomy, the patient was given prophylactic vaccination (Streptococcus pneumoniae, Haemophilus influenza type $b$ and Neisseria meningitidis,) and was started on a sixmonth course of prophylactic penicillin.
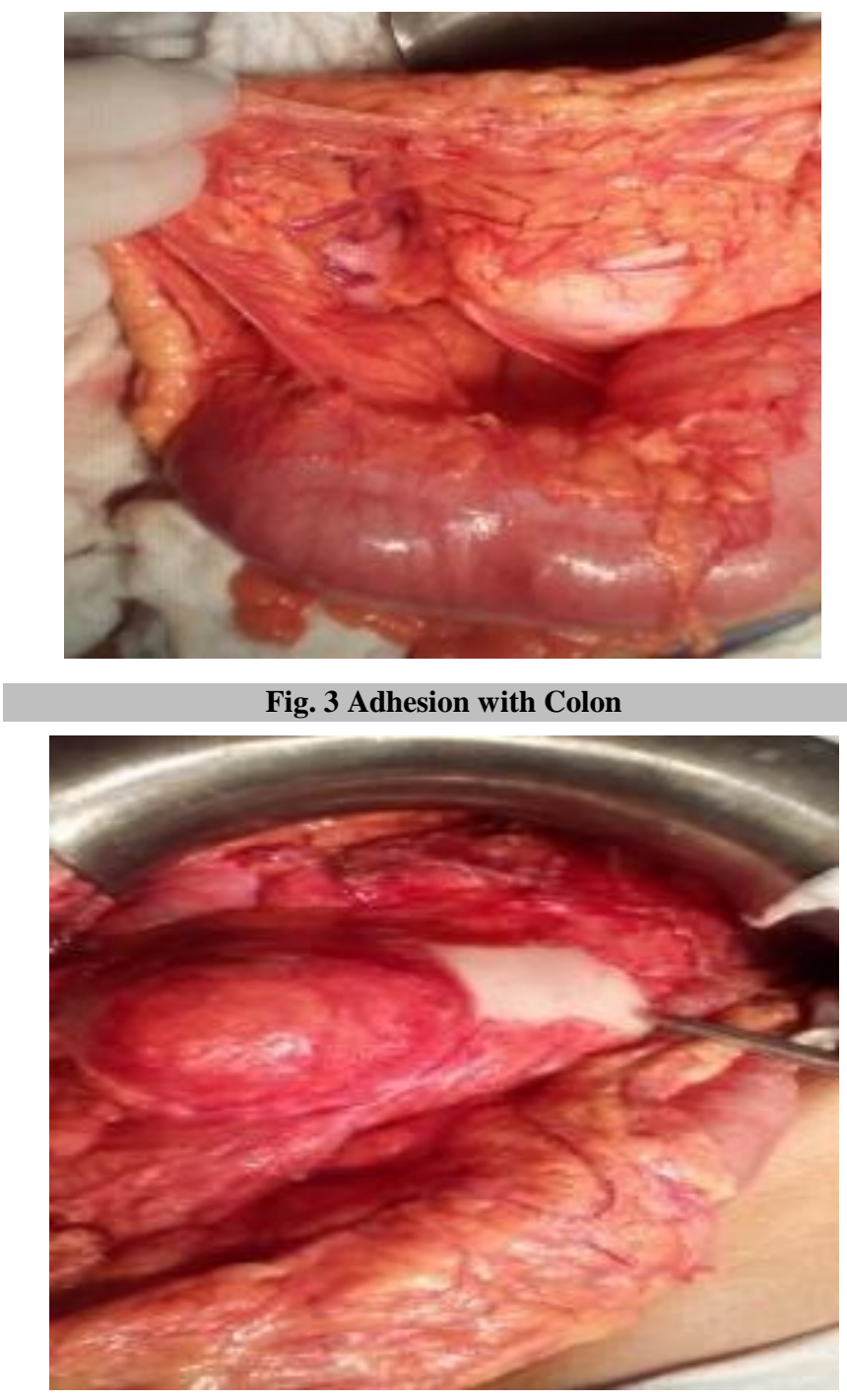

Fig. 4 Adhesions with Diaphragm
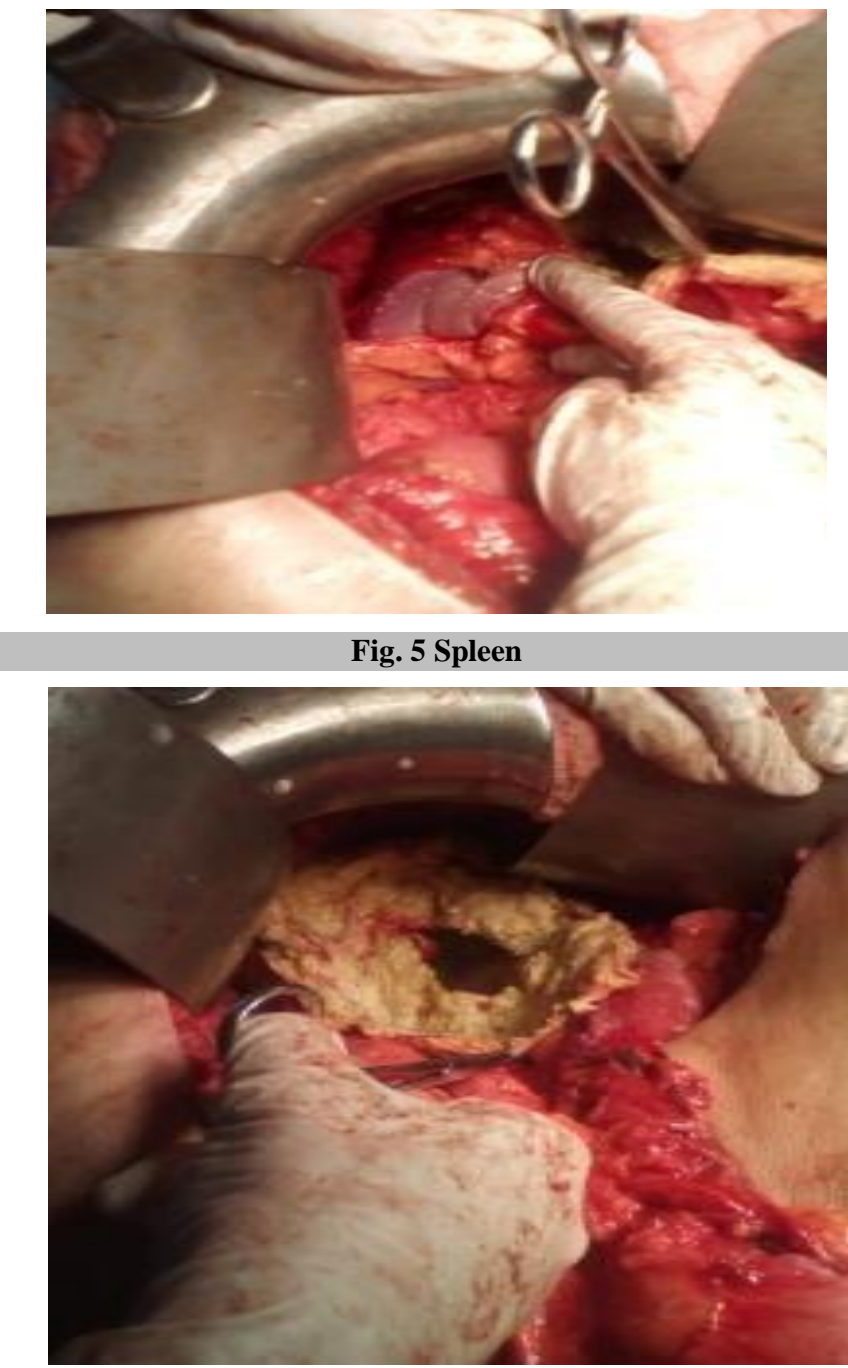

Fig. 6 Cavity of Cyst

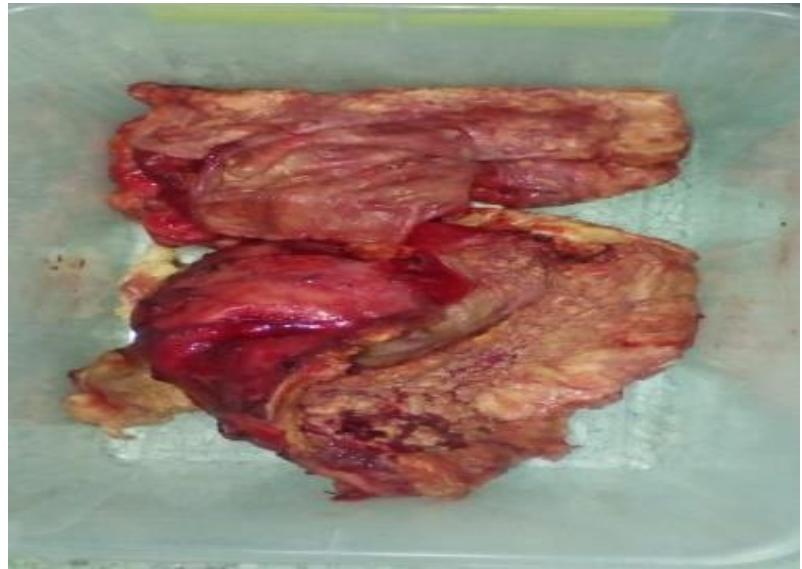

Fig. 7 Total Splenectomy with Large Cyst 


\section{Discussion}

The treatment of hydatid cyst of spleen is essentially surgical.

Total splenectomy has the advantage of suppressing the parasitized organ and avoiding secondary recurrences, as for our patient, it can be difficult due to cyst-visceral adhesions. The resection of the protruding dome has the advantage of being a benign, almost always feasible, since the hydatid cyst is accessible to the surface of the spleen.

On the other hand, it leaves periyste in place, which can be the seat of residual cavity and postoperative infection.

The pathway depends first on the location of the splenic cyst and on the association with other cystic sites.

However, if the splenic cyst is infected,(4) have metastatic implantations to adjacent organs or the locations and size do not allow safe resection, conservative surgical technic such as partial splenectomy, cyst enucleation, deroofing of the cyst with omentoplasty or external drainage may be used.

Many trials (1) are usually made for conserving the spleen, so as to avoid overwhelming post splenectomy sepsis (OPSI).

Partial splenectomy carries a risk of poor vascular control when incising the splenic tissue while unroofing the cyst wall leaving behind a residual cavity carries the risk of postoperative infection.

For the above reasons and the possibility of multiple splenic cysts, total splenectomy should be the method of choice, (5) especially in the presence of a communication between the spleen and nearby organs, such as the stomach, colon and diaphragm $(2,3)$.
Laparoscopic approach has also been advocated for uncomplicated hydatid cyst of the spleen.

\section{Conclusion}

Hydatid disease should be considered in the differential diagnosis of all cystic masses in the spleen especially in the geographical regions where the disease is endemic.

The splenic hydatid cyst may become a challenging surgical problem. Preoperative evaluation should be carried out carefully. Computerised tomography scan is the most sensitive investigation for diagnosis. The anatomical relations of splenic hydatid cyst should be demonstrated before surgery on account of varied presentations. Although the management must be individualized for each.

\section{References}

1. Tarcoveanu E., Plesa A., Danila N., Lupascu C., Cotea E., Negru R. (2002). Splenic hydatic cyst, Observations upon 38 cases of splenic echinococcosis. Revista Medico- Chirurgicala a Societatii de Medici Si Naturalisti Din Iasi, 107:311-315.

2. Lippitt W.H., Akhavan T., Caplan G.E. (1967). Epidermoid cyst of the spleen with rupture and inflammation. Archives of Surgery, 95(1):74-78.

3. Teke Z., Yagci A.B., Atalay A.O., Kabay B. (2008). Splenic hydatid cyst perforating into the colon manifesting as massive lower gastrointestinal bleeding: an anusual presentation of disseminated abdominal echinococcosis. Singapore Medical Journal, 49:113- 116.

4. Hoffman E. (1957). Non-parasitic splenic cysts. The American Journal of Surgery, 93(5):765-770.

5. Berrada S., Ridai M., Mokhtari M. (1991). Hydatid cysts of the spleen splenectomy or conservative surgery. Ann Chir, 45:434 436. 